\title{
A Cross Sectional Descriptive Study of Etiology and Clinical Pattern of Liver Abscess : 50 Cases
}

\author{
Joyabrata Das ${ }^{1 *}$ \\ Subash Majumdar ${ }^{2}$ \\ Subrata Das ${ }^{3}$ \\ Saiem Nurul Anwar ${ }^{1}$ \\ Hossain Ahmed' \\ Istiaque Hossain \\ 'Department of Medicine \\ Southern Medical College \& Hospital \\ Chittagong, Bangladesh. \\ ${ }^{2}$ Department of Radiology \\ Chittagong Medical College \& Hospital \\ Chittagong, Bangladesh. \\ ${ }^{3}$ Department of Ophthalmology \\ Chittagong Medical College \& Hospital \\ Chittagong, Bangladesh.
}

${ }^{*}$ Correspondence to:

\section{Dr. Joyabrata Das}

Associate Professor

Department of Medicine

Southern Medical College \& Hospital

Chittagong, Bangladesh.

Mobile: +88-01819318334

E-mail:drdasjb@g mail.com

\begin{abstract}
Background: The liver is the organ most subjected to the development of abscesses and made up $48 \%$ of all visceral abscesses' and $7 \%$ of all intra abdominal abscesses. Liver abscess should be suspected when there is a combination of fever, leucocytosis, constitutional symptoms, and pain in the right upper quadrant, and tenderness over the liver or right lower rib cage. The liver is affected by a number of local and disseminated infections; their frequency and types vary considerably around the world. Parasitic disorders are more prevalent in developing countries. Methods: It was a cross-sectional descriptive study. The cases were taken from the admitted patients of Medicine unit of $\mathrm{SMCH}$, Chittagong. The study was conducted over a period of one year with a sample size of 50 patients and sample was taken by purposive sampling. Results: Among 50 patients $40(80 \%)$ were diagnosed as ALA and $10(20 \%)$ were diagnosed as PLA. ALA cases are common in 21-30 years age group \& PLA cases are more common in $>50$ years of age group. Majority of the cases were from rural area. Common clinical features were fever, abdominal pain, nausea and tender hepatomegaly. Diarrhea was present on admission in $7(15.9 \%)$ patients of ALA. Anaemia was common in both but polymorphonuclear leucocytosis was moderate to severe in PLA. Microscopic examination of stool samples for E. Histolytica trophozoites was positive in $3(7.5 \%)$ cases and cysts in $4(10 \%)$ cases. Nine patients had right sided pleural effusion. Conclusions: Clinical features are common in both ALA and PLA. Liver abscesses are more common in men and more prevalent in rural areas. ALA more commonly occurs in 21-30 years age group but can occur at any age. Pyogenic abscess is more common in older age group (>50 years) and E. Coli is the commonest organism.
\end{abstract}

Key words: Amoebic liver abscess; Pyogenic liver abscess; Anchovy sauce; Escherichia Coli; Entamoeba Histolytica.

\section{INTRODUCTION}

The liver is the organ most subjected to the development of abscesses and made up $48 \%$ of all visceral abscesses' and $7 \%$ of all intra-abdominal abscesses ${ }^{1-2}$. Liver abscess should be suspected when there is a combination of fever, leucocytosis, constitutional symptoms, and pain in the right upper quadrant or right lower chest, and tenderness over the liver or lower rib cage ${ }^{3}$. The liver is affected by a number of local and disseminated infections; their frequency and types vary considerably around the world. Parasitic disorders are more prevalent in developing countries, especially those in tropical and subtropical regions ${ }^{4}$. Pyogenic liver abscesses are important because they are potentially curable, inevitably fatal if untreated and readily overlooked ${ }^{5}$. Amoebic and pyogenic abscess share many clinical, laboratory and imaging feature; at the same time they differ sharply in some respect, particularly with regard to epidemiology and treatment. Differentiation is essential for effective treatment ${ }^{3}$. Amoebic serologic testing gives positive results in more than $95 \%$ of cases; thus a negative result helps to exclude this diagnosis ${ }^{1}$. Amoebic abscess of the liver is one of the terminations of amoebic hepatitis, which in turn is a complication of amoebic dysentery not so very uncommon ${ }^{6}$. The World Health Organization estimates that annually 50 million cases of amoebic colitis and liver abscess and 100,000 deaths result from E.Histolytica infection and the major cause of mortality is amoebic liver abscess ${ }^{7,8,9}$. 
In the majority of cases, the hepatic complication appears after about one to three months of the disappearance of the dysenteric attack. Cases have been reported where liver abscesses have developed after a lapse of months or years. It is rather a peculiar feature that patients suffering from acute dysenteric symptoms do rarely present hepatic complications. Only in a small number of cases both the intestinal and the liver are involved at the same time, and the cases are invariably fatal. ${ }^{10}$ A moebic liver abscess, if untreated, leads to fatal outcome with mortality rate of $7-14 \%{ }^{11}$. In $A L A$, the presence of bacteria does not indicate pyogenic abscess ${ }^{12}$. Pyogenic liver abscess are focal areas of infection within the hepatic parenchyma, these abscesses may be resulted from invasions by a variety of bacteria. Pyogenic liver abscess should be considered in any patient who has presumptive or definite evidence of septicemia in association with biochemical abnormalities of the liver ${ }^{4}$ The patient with a pyogenic liver abscess usually has a shorter duration of symptoms, is more ill, and often has a history of co-existing or pre-existing diseases ${ }^{13}$. The diagnosis of amoebic liver abscess is sometimes difficult and the key to successful diagnosis and treatment of pyogenic liver abscess is identification of the organism in the abscess. In comparison, the aspirate of amoebic liver abscess is a mixture of sloughed liver tissue and blood. The aspirate is chocolate brown in colour and thick in consistency (the so called "anchovy-sauce"). The trophozoites of E.Histolytica are not generally found in freshly aspirated liver-pus but appear in the escaping "pus" four or five days after the initial evacuation ${ }^{12}$. In aspirates, identification of E. Histolytica is possible only a few cases. Pyogenic abscess aspirate is cream coloured, light tan or green-tinged and often has the putrid odor of anaerobes, contain a heavy neutrophilic inflammatory exudate with nuclear debris and almost invariably showed culture positive ${ }^{14}$. Common organisms are Klebsiella Pneumoniae, E. Coli, Streptococcus, bacteroides, and Enterococcus ${ }^{15}$. Liver abscesses are very common in our community both in rural and urban area, complications from liver abscesses are also very common. So it is important to study the aetiology and clinical pattern of liver abscess in our community.

\section{MATERIALS AND METHODS}

It was a cross-sectional descriptive study. The cases were taken from the admitted patients of different medicine units of $\mathrm{SMCH}, \mathrm{Chittagong}$. The study was conducted over a period of one year from J anuary 2009 to December 2010. The study was done on a total of 50 patients suffering from liver abscess. Sample was taken by purposive sampling. Aims of the study were:

1) To know the clinical presentation of Liver abscess patients.

2) To identify the cause of Liver abscess

3) To find out the complications.

\section{A . Inclusion C riteria}

1. Patients above 13 years of age (both male and female). 2 . Detected by USG as having liver abscess. 3. Liver abscess confirmed by aspiration of pus. 4. Patients were selected from the admitted patients in medicine units of SM CH.

\section{B. Exclusion criteria}

1. A ge below 13 years (both male and female) 2) Cases where aspiration of pus was not possible. 3) Extremely ill patient. 4) Those who did not give consent.
Elaborate history was taken from all patients and they were examined meticulously. After clinical diagnosis relevant investigations (as far as feasible in study institute i.e.-SM CH) were done. Liver aspirate, blood and stool were collected from all the 50 patients.

Aspiration of liver abscess - A spirate samples were collected in the ward under strict aseptic precautions through the right $8^{\text {th }}$ intercostal space or through right sub-costal region by lumber puncture needle. USG guidance was taken in all the cases. In all cases pus was aspirated and if aspiration of pus couldn't be possible, then these patients were not included in the study protocol. After aspiration, The color of aspirate was noted in naked eye and also sent for culture and sensitivity and microscopic examination for trophozoite of amoeba, cytology and Gram staining to identify the organism with all aseptic precaution into the pathology department of $\mathrm{SMCH}$. Organism was identified by lactose fermentation and by microscopic and macroscopic physical appearance. Though culture of pus does not reveal any amoeba, a negative culture of pus supported by naked eye and microscopic finding of pus is taken as a case of amoebic liver abscess in this study as serum antibody against amoeba (serological test) cannot be done in this institute. Pus culture positive cases are considered as pyogenic liver abscess.

\section{Investigations}

1) Blood for TC, DC, Hb\%, ESR 2) Stool R/M/E 3) X-Ray chest P/A view 4) USG of hepatobiliary system 5) Culture/Sensitivity of pus 6) Microscopic examination of liver aspirate.7) Blood sugar-fasting and two hours after breakfast.

\section{Data recording and Analysis}

All relevant information was collected by interviewing patients or their guardians and recorded in a pre-designed data sheet. Reports of investigations were also preserved. All cases were purposively selected. Z test used for tests of Significance.

\section{RESULTS}

A total of 50 clinically and ultrasonographically diagnosed liver abscess patients of 13-75 years of age were studied, 41 were males; 40 were ALA and 10 PLA (Table 1). All 50 cases were from rural area. Symptoms at presentation were depicted in table 2. Ten patients were diabetic, 2 had history of appendicitis and 3 had gallbladder stones. Routine haematological tests revealed leucocytosis in 44 cases and mild to moderate anemia in 42 cases (Table 3). A ccording to USG report 40 patients were having single abscess and the remainder had multiple abscesses. The largest size is $19 \times 16 \mathrm{~cm}$ and the smallest one $5.5 \times 4.3 \mathrm{~cm}$. The average size is having $8-10 \mathrm{~cm}$ in diameter. On aspiration as much as $800 \mathrm{ml}$ pus was aspirated and the lowest amount found in one case was $30 \mathrm{ml}$. But on an average 120-150 ml was aspirated. In naked eye examination colour of 40 cases was chocolate or brown and in the rest 10 cases it was whitish or grayish. On microscopic examination of pus trophozoite of amoeba were found in 11 cases. On the $C / S$ report of pus only in 10 cases culture was positive and organisms were found. E.Coli is the commonest organism in PLA (Tables 4 and 5). Of the 10 culture positive cases all were having multiple abscesses but some multi abscesses cases were $\mathrm{C} / \mathrm{S}$ negative. In X-ray chest P/A view only in 3 cases (having large abscess) right dome of diaphragm was slightly elevated but in all other cases it was normal. In nine cases there was associated mild pleural effusion also on the right side. Stool R/M/E in three cases revealed trophozoite of amoeba and in four cases cyst of amoeba. 
Table 1: A ge \& Sex distribution of patients with amoebic and pyogenic liver abscess

\begin{tabular}{|c|c|c|c|c|c|c|}
\hline \multirow[t]{2}{*}{ Age in Years } & \multicolumn{2}{|l|}{ ALA } & \multirow{2}{*}{$\begin{array}{c}\mathrm{n}=40 \\
\text { Total }\end{array}$} & \multirow{2}{*}{$\frac{\text { PLA }}{\text { Male }}$} & \multirow[b]{2}{*}{ Female } & \multirow{2}{*}{$\begin{array}{l}\text { n=10 } \\
\text { Total }\end{array}$} \\
\hline & Male & Female & & & & \\
\hline$<20$ & 4 & 0 & $4(10.0)$ & 0 & 0 & 0 \\
\hline $21-30$ & 11 & 2 & $13(32.5)$ & 0 & 0 & 0 \\
\hline $31-40$ & 8 & 3 & $11(27.5)$ & 0 & 0 & 0 \\
\hline $41-50$ & 5 & 2 & $7(17.5)$ & 2 & 0 & $2(20.0)$ \\
\hline $51-60$ & 1 & 0 & $1(2.5)$ & 4 & 0 & $4(40.0)$ \\
\hline$>60$ & 2 & 2 & $4(10.0)$ & 4 & 0 & $4(40.0)$ \\
\hline Total & $31(77.5)$ & $9(22.5)$ & $40(100.0)$ & 10(100) & $0(0)$ & $10(100.0)$ \\
\hline
\end{tabular}

(Figures in Parenthesis indicate Percentage)

Table 2: Symptoms at the time of presentation

\begin{tabular}{lcc} 
& & Patients with liver abscess \\
\cline { 2 - 3 } & AL $\mathbf{A}(\mathbf{n}=\mathbf{4 0})$ & PL $\mathbf{A}(\mathbf{n = 1 0})$ \\
Fever & $40(100)$ & $10(100)$ \\
Pain in the right hypochondriac region & $36(90.0)$ & $2(20.0)$ \\
Diffuse abdominal pain & $6(15.0)$ & $4(40.0)$ \\
Nausea & $18(45.0)$ & $2(20.0)$ \\
Vomiting & $5(12.5)$ & $3(30.0)$ \\
Diarrhoea & $7(17.5)$ & $0(0)$ \\
Chest pain/Cough & $6(15.0)$ & $0(0)$ \\
Weight loss & $1(2.5)$ & $3(30.0)$ \\
\hline
\end{tabular}

(Figures in Parenthesis indicate Percentage)

$\mathrm{P}<0.001$ : In Fever, Right hypochondriac pain, $\mathrm{N}$ ausea; $\mathrm{P}<0.05$ : In diarrhea, $C$ hest pain, $\mathrm{A}$ bdominal pain

Table 3: Haematological findings among the liver abscess patients.

\begin{tabular}{lcc} 
Haematological findings & AL $\mathbf{A}(\mathbf{n}=\mathbf{4 0})$ & PL A(n=10) \\
A naemia & $32(80.0)$ & $\mathbf{1 0}(\mathbf{1 0 0})$ \\
Leucocyte count (thousands/cumm) & & \\
$<11.0$ & $06(15.0)$ & $0(0)$ \\
$11.1-15.0$ & $30(75.2)$ & $4(40.0)$ \\
$>15.0$ & $04(10.0)$ & $6(60.0)$ \\
Percentage of neutrophils & & $0(0)$ \\
$<70$ & $5(12.5)$ & $4(40.0)$ \\
$71-80$ & $34(85.0)$ & $6(60.0)$ \\
$>80$ & $1(2.5)$ & \\
\hline
\end{tabular}

(Figures in Parenthesis indicate Percentage)

$\mathrm{P}<0.001$ : In A naemia

Table 4: Microscopic findings of ALA \& PLA aspirate

\begin{tabular}{|c|c|c|c|c|c|c|c|c|c|c|c|}
\hline \multirow{2}{*}{$\begin{array}{l}\text { Liver } \\
\text { aspirates } \\
\text { n=50 }\end{array}$} & \multicolumn{3}{|c|}{ Pus cells } & \multicolumn{3}{|c|}{ R BC } & \multicolumn{3}{|c|}{ Necrotic debris } & \multirow{2}{*}{$\begin{array}{c}\text { E. } \\
\text { Histolytica } \\
\text { (Trophoz } \\
\text { oite) }\end{array}$} & \multirow{2}{*}{$\begin{array}{l}\text { Organism } \\
\text { in G ram } \\
\text { stain }\end{array}$} \\
\hline & $\mathbf{F}$ & M & $\mathbf{P}$ & $\mathbf{F}$ & M & $\mathbf{P}$ & $\mathbf{F}$ & M & $\mathbf{P}$ & & \\
\hline $\begin{array}{l}\text { A } \mathrm{A} \\
\mathrm{n}=40 \\
\text { (C/S negative) }\end{array}$ & $\begin{array}{r}30 \\
(75)\end{array}$ & $\begin{array}{l}10 \\
(25)\end{array}$ & 0 & $\begin{array}{c}35 \\
(87.5)\end{array}$ & $\begin{array}{c}3 \\
(7.5)\end{array}$ & $\begin{array}{c}2 \\
(5.0)\end{array}$ & 0 & $\begin{array}{c}5 \\
(12.5)\end{array}$ & $\begin{array}{c}35 \\
(87.5)\end{array}$ & $\begin{array}{c}11 \\
(27.5)\end{array}$ & 0 \\
\hline $\begin{array}{l}\text { PLA } \\
n=10 \\
\text { (C/S positive) }\end{array}$ & 0 & $\begin{array}{c}1 \\
(10.0)\end{array}$ & $\begin{array}{c}9 \\
(90.0)\end{array}$ & $\begin{array}{c}5 \\
(50.0)\end{array}$ & $\begin{array}{c}5 \\
(50.0)\end{array}$ & 0 & $\begin{array}{c}10 \\
(100)\end{array}$ & & & & $\begin{array}{c}10 \\
(100)\end{array}$ \\
\hline
\end{tabular}

F-Few, M-M oderate, P-Plenty (Figures in Parenthesis indicate

Percentage) $P<0.05:$ In E. Histolytica 
Table 5: Rate of isolation of bacteria from liver aspirate of patient with PLA

\begin{tabular}{lc} 
Organisms isolated & PL A $\mathbf{n}=\mathbf{1 0}$ \\
E.Coli & $5(50)$ \\
Proteus & $3(30)$ \\
Pseudomonas & $1(10)$ \\
Klebsiella & $1(10)$ \\
\hline
\end{tabular}

(Figures in Parenthesis indicate Percentage)

$\mathrm{P}<0.05$ : In E. Coli, Proteus.

\section{DISCUSSION}

The liver is the organ most subjected to the development of abscess and made up $48 \%$ of all visceral abscesses. The patient under study having a liver abscess was confirmed by USG and aspiration of pus by ultrasonic guidance. This study was performed among 50 patients aged from 13 to 75 years. In all patients blood sample and liver aspirate were studied to find out the aetiology of liver abscess. Differentiation of ALA \& PLA was done on the evidence of naked eye examination and culture of pus and microscopic finding of liver aspirate (e.g. trophozoite of amoeba, cytology and organism in gram staining). In ten cases pus culture was positive and also the color of pus in these cases was greyish white or creamy. In our study all 50 aspirates were examined with gram staining. Ten aspirates showed organisms and all these 10 samples were positive for aerobic culture. Our findings strongly correlated with Barnes et al. (1987) in USA who stated that the presence of bacteria on gram's stain or culture of the aspirates might be the indicator of PLA ${ }^{16}$. In the other 40 cases culture of pus did not yield any organisms. In these cases the colour of aspirate was chocolate, reddish brown. Among these 40 cases, trophozoites of E. Histolytica were detected microscopically in 11 cases (27\%). In Durban, Gathiram et al. (1984) reported $21 \%$ cases were positive for $\mathrm{E}$. Histolytica trophozoites in aspirate. ${ }^{17}$ M ohajan \& Ganguly (1980) in India stated only $15.2 \%$ of liver aspirate showed the presence of $E$. Histolytica and in Bangladesh Rab et al. (1967) reported E. Histolytica trophozoites in all aspirates of complicated cases ${ }^{18-19}$. M oderate to few pus cells \& plenty necrotic debris found in microscopy of aspirates in culture negative cases, while plenty of pus cells were present in the 10 culture positive cases. Few RBC were found the most of the liver aspirates and moderate to plenty RBC were found in cases, probably due to traumatic injury during percutaneous aspiration. Chatterjee (1984) in India and Rab et al. (1966) ${ }^{2}$. In Bangladesh liver aspirates in ALA were described as "anchovy-sauce pus" because of chocolate brown colour \& thick consistency. Reynolds (1992) in USA showed degenerated liver cells, a few RBC and occasional leucocytes in A LA aspirates and many neutrophils in PLA aspirates ${ }^{3}$. So with the evidence of chocolate or reddish brown colour \& negative culture of pus, plenty necrotic debris and few pus cells in microscopy and no organism in gram staining, these 40 cases were screened as ALA. Of the 50 patients studied relatively higher number $13(26 \%)$ of cases were found in age group of 21-30 years, followed by $11(22 \%)$ in age group $31-40$ years. Males predominated $41(82 \%)$ than females. In Bangladesh, khan et al. (1991) reported $31.1 \%$ cases of A LA in age group 21-30 years. ${ }^{20}$ On the other hand among PLA patients all the 10 patients were of age 50 or more. Petri et al, (1999) stated roughly $90 \%$ of A LA patients were young adult male around 30 years and patient with PLA usually belongs to older generation, aged more than 50 years ${ }^{21}$. So, in this study both the PLA and A LA age group correlates with other study. In our study, only in 10 cases the culture of pus yielded growth of organism. In these cases PLA, the predominant organism were $\mathrm{E}$. Coli $5(50 \%)$ and other organism Proteus 3(30\%) Pseudomonas and K lebsiella each $1(10 \%)$. These studies are in agreement with $M$ oore et al. (1993) in U.K, M CD onald (1984) in A ustralia, Sherlock et al. (1993) in U.K, they found E. Coli as the Commonest organism 22-24. In contrast Yeah et.al (1997) in Singapore, reported Klebsiella Pneumoniae as the commonest organism in $P L A^{25}$. In this study no fungal species were detected. M cdonald (1984) in A ustralia reported occasional liver abscesses caused by Candida A lbicans ${ }^{23}$. Out of the $40 \mathrm{ALA}$ patients in majority of cases $80 \%$ the colour of the aspirate was chocolate colour where as in $10(20 \%)$ PLA cases it was whitish or creamy colour. Chatterjee (1994) in India and Rab et al. (1966). In Bangladesh described liver aspirates as "anchovy-sauce pus" because of chocolate brown colour and thick consistency. Reynolds (1992) in USA showed degenerated liver cells, a few red blood cell and occasional leucocytes in ALA aspirates and many neutrophils in PLA aspirates which was cream coloured, light tan or green tinged. All 50 cases of this study came from rural area and most of them from remote areas. In this study fever was common as it was present all the cases $(100 \%)$ followed by abdominal pain. So, fever was the presenting symptoms of both the amoebic and Pyogenic liver abscess. Pain in the right hypochondriac region was found in 40 cases (80\%). In the rest 10 cases there was diffuse abdominal pain. Weight loss was found only in 4 cases (8\%). Weight loss may be due to the chronicity of the disease and in some patients due to inadequate intake of food as a result of nausea, vomiting and anorexia. Nausea was found in 20 Cases $(40 \%)$ and vomiting in 8 cases $(16 \%)$. K han et al. $(1997)^{20}$ in Bangladesh gave little lower values for symptoms such as fever (75.55), pain in the abdomen and epigastrium (71.1\%) weight loss (26.7\%) in A LA patients. Most of the liver abscesses were in the right lobe $(74 \%)$ and probably few were higher enough to give some of the chest findings. Only in 9 patients there was right sided pleural effusion found both in chest X-ray and USG. This finding are in agreement with the observations of petri et al. (1997) in B angladesh, reported 33.3\% cases of A LA had cough and $\mathrm{Rab}$ et al. (1967) reported abnormal chest findings in 3 $(75 \%)$ atypical cases of ALA patients. Whereas, Stenson et al. (1983) in USA, reported about 50\% PLA patients had chest findings. On abdominal examination mild to moderate tenderness on palpation was present in all the 50 cases i.e. both in ALA and PLA cases. But in 2 patients the abdomen was highly tender and in USG of those 2 patients there was multiple large abscesses. In 40 cases (80\%) tenderness was confined only in right hypochondriac region but in other $10(20 \%)$ cases there was diffuse abdominal tenderness. The liver was found enlarged on palpation in $30(60 \%)$ cases. These findings correlate with other studies, which states that clinically hepatomegaly is found in $50 \%$ or more than $50 \%$ cases but right hypochondriac tenderness is found in almost all cases. In present study anemia was present in ALA $(80 \%)$ and PLA $(100 \%)$ patient. In ALA mild to moderate leucocytosis occured $(75 \%)$ at the time of diagnosis. Whereas moderate $(40 \%)$ to severe $(60 \%)$ polymorphonuclear leucocytosis was common in PLA (100\%) patients.Barnes et al. (1987) ${ }^{16}$ in USA reported anaemia in both ALA $(70 \%)$ and PLA $(70 \%)$ patient and similar number of cases had leucocytosis and with a marked shift to the left ( $>89 \%$ neutrophils) in PLA. In Bangladesh, $\mathrm{K}$ han et al. (1997) showed $26.7 \%$ polymorphonuclear leucocytes in Rab et al. (1967) reported leucocytes in $4(100 \%)$ complicated ALA cases. But in India, Aikat et al. (1978) observed normal leucocytes count in 27\% ALA patients and leucopoenia in $(5.9 \%)$ cases $^{23}$. In our study only $12.5 \%$ cases showed normal leucocytes count and none showed leucopenia. In present study only few patients of ALA (17.5\%) and none 
of the PLA patients had diarrhoea at the time of hospitalization. The findings are in contrast with Barnes et al. (1987) who reported that diarrhoea occurred equally frequently in both A LA (7\%) and PLA (6\%) and Reynolds (1992) reported mild to moderate diarrhoea in about one-third of patients with both type of abscesses in USG ${ }^{16}$. Stool samples of all 50 liver abscess patients were examined microscopically specially for $E$. Histolytica. In A LA trophozoites of E. Histolytica were found in $3(7.5 \%)$ cases and cysts in $4(10 \%)$ cases. Our microscopic findings are much lower than the other reported values. Probably most of these cases of liver abscess were treated with metronidazole before admission into hospital i.e. before collection of stool samples, which eliminated the parasites from the gut. E. Histolytica in stool was found in $18.5 \%$ in India (Baveja et al. 1992), 15\% in UK (Sherlock, 1993) and 18\% in USA (Petri, 1994) at the time of diagnosis of amoebic liver abscess. In 6(15\%) A LA patients and in 3(30\%) PLA patient right sided pleural effusion was found. Back et al found pleural effusion in $4 \%$ of cases in a study of both ALA and PLA. In this study higher percentage of PLA cases had pleural effusion, which may be due to small sample size.

\section{CONCLUSIONS}

Clinical features are common in both ALA and PLA. Though it is easy to diagnose a case of liver abscess clinically, it is not possible to differentiate between ALA \& PLA by clinical features. Liver abscess is more common in men. It is more prevalent in rural areas. A LA more commonly occurs in 21-30 years age group but can occur at any age. Pyogenic abscess is more common in older age group ( $>50$ years). E. Coli is the predominant organism in PLA. It is a hospital based study so it does not represent the total community. Sample was purposively selected so there was a chance of biasness.

\section{DISCLOSURE}

The study was ethically reviewed and there is no conflict of interest.

\section{REFERENCES}

1. Zaleznik F, Dori, Kasper L, Dennis, Intra-abdominal Infections and abscess, $15^{\text {th }}$ edition, In Fauci AS, B raunwald E, Isselbacher KJ, Wilson JD, M astin J B, K asper DL, Huser SL, L ongo DL (eds) Harrison's Principles of internal M edicine, M cG raw-Hill Companies, Inc. USA, 2002; 752.

2. Grant SW, Wilson. SE Intra-abdominal abscess: Diagnosis and management. In kelly W N (ed. in chief) Textbook of Internal M edicine, $3^{\text {rd }}$ ed. Lippincoyy-Raven Publisher, Philadel phia, N ew York, 1997; 1556-1557.

3. Reynolds TB, Liver A bscess, In Neil Kaplowitz, Liver and Biliary Diseases. Williams \& Wilkins (Pub), Baltimore, USA. 1992; 415-420.

4. Maddrey WC. Parasitic, B acterial, Fugal, and Granulomatous Liver disease. In Bannett J C; Plum F, Cecil Textbook of M edicine, $20^{\text {th }}$ edition, W B Saunders Company, Philadel phia. 1996; 781-785.

5. Finlaysons NDC, B ouchier IA D. Diseases of the liver and biliary system. In Edwards CRW, Dauchier IAD, Haslett C, Chivers E (ed) Davidsons Principles and Practice of M edicine, $7^{\text {th }}$ edition, Churcill Living stone Inc. ON ewyork, NK 10011, 1996; 483-545.

6. J ohnson AG, Shields R. The liver. In Baily \& Love's (ed) Short Practice of Surgery. Revised by M ann CV, Russel RCG, H.K. L ewis \& Co.Ltd. 1985; Chapman \& Hill, 1995.

7. Munoz M DL, Lamoy E. Leon G, Tover R, Perez-Garcia J, Torre M De La, M urueta E and Bernal RM. A ntigen in Electon-Dense Granules from Entamoeba hietolytica as possible marker for pathogenecity. J Clin Microbiol 1990; 28(11): 2418-24.

8. World Health Organization (G eneva, 26-28 M arch 1980), Diarrheal disease control program, Epidemiology, pp. 6-11.

9. Dodson JM, Lenkowski PW, Eubank AC, J ackson TFGH, Napondano J, Lyerly DM , L ockhart LA, M ann BJ, and Petri W A, Infection and immunity mediated by carbohydrate recognition domain of the Entamoeba Histolytica GallGalNA c lectin. J Inf Dis 1999; 179: 460-6.

10. Ellen and Stanley, SL, J r. Protozoa, A moebiasis. Gstronterol. Clin. N orth. A m. 1988.

1I. Walsh JA. Transmission of Entamoeba Histolytica infection. In Ravdin JI (ed) A moebiasis: Human infections by Entamoeba Histolytica. John Wiley and Sons, USA, 1988; 106-119.

12. Chatterjee KD. Parasitology (Protology and Helminthology) in relation to Clinical M edicine. $12^{\text {th }}$ (edn). Chatterjee M edical Publishers. Calcutta, India, 1994; 19984.

13. Sepulveda B, M anzo NTG. Clinical manifestations and diagnosis of amoebiasis. In Palomo-M A (ed) A moebiasis. 1986; 169-188.

14. Palomo-M A and Cantellano EM . Intestinal A moebae. In Cox PEG, K reier JP, Wakelin D (ed) Topley \& Wilsons M icrobiology and M icrobial Infections. Parasitology 1998; 5: 157-177.

15 Jalan KN and M itra Tk. A moebiasis in the developing world. In Ravdin J (ed) A moebiasis: Human Infections by Entamoeba Histolytica. John Wiley and Sons, USA, L ondon, 1988; 535-555.

16. Barnes PF, DeCock KM , Reynolds TN, Rails PW. A case of A moebic and Pyogenic A bscess of the Liver. In M edicine 1987; 66(6): 472-483.

17. Gathiram Y, Simjee AE, B hamje A, J ackson TFHG, Pillary L V, A nderson CB. Concomitant and secondary bacterial infection of the pus in hepatic amoebiasis. South Afr M ed J 1984; 65: 951-53.

18. Mahajan RC. and Ganguly Nk. A moebic antigen in immunodignosis and prognosis of amoebic liver abscess, Trans. Royal Soc. Trop. M ed. Hyg 1980; 74(3): 300-302.

19. Rab SM , A lam M N, Huda AN and Yee A. A moebic liver abscess: Some unique presentations. A J M ed 1967; 43: 811-16.

20. K han M , A kther A, M amun A A, M ahmud TAK, A hmed KU. A moebic liver abscess: clinical profile and therapeutic response. Bang J MED 1991; 2:32-38.

21. Petri W A J r. and Singh U. Diagnosis and management of amoebiasis. Clin Inf Dis 1999; 29: 1117-25.

22. M oore-Gillin JC, Eykyn S, Phillips I. M icrobiology of pyogenic liver abscess. Papers and short reports. B MJ 1981; 283: 819-21.

23. MCDonald MI. Pyogenic liver abscess: Diagnosis, Bacteriology and Treatment. Eur J Clin Microbiol 1984; 3: 500-509.

24. Sherlock S, Dooley J. The liver in infection. In Sherlock S, D ooley J. (ed) Disease of the liver and biliary system. B lackwell Scientific Publications, London. $11^{\text {th }}$ edition, 495.

25. Yeah HM , Kirn WH, Shin SK, Chun WH, Kong JK, Park IS. The changing patterns of liver abscess during the past 20 years-a study of 482 cases. Yeosec M ed J 1993; 34(0): 340-350. 